ITP-SB-95-30

hep-ph/9508358

\title{
Soft Gluon Resummation
}

\author{
George Sterman \\ Institute for Theoretical Physics \\ State University of New York, Stony Brook, NY 11794-3840
}

\begin{abstract}
I discuss some of the basic techniques and results of soft gluon resummation, and their applications to scattering at collider energies.
\end{abstract}

\footnotetext{
${ }^{1}$ Based on a talk presented at the 10th Topical Workshop on Proton-Antiproton Collider Physics, Fermilab, May 9-13, 1995
} 


\section{Varieties of Factorization and Evolution}

With the extraordinary data from the Tevatron of the last runs, our knowledge of large momentum transfer processes has taken a qualitative step forward. The demands on theory are now much more stringent, and afford tests of QCD concepts that were not previously practical. In particular, $\mathrm{TeV}$ energies allow for many two-scale processes where both scales are much larger than a $\mathrm{GeV}$, and hence may be amenable to perturbative treatment. For instance, $\alpha_{s}(t) \ln (s / t)$ may be a relatively large number even for $\alpha_{s}(t)$ small, necessitating a resummation of powers $\left(\alpha_{s}(t) \ln ^{2}(s / t)\right)$ and $\left(\alpha_{s}(t) \ln (s / t)\right)$ to all orders in perturbation theory.

In the following, I shall review the basic techniques used to study two-scale inclusive cross sections at low parton density. For such process factorization in terms of single-parton densities holds,

$$
\sigma_{H}(p, q)=\sum_{i} \int d x^{\prime} \hat{\sigma}_{i}\left(x^{\prime} p, q\right) \phi_{i / H}\left(x^{\prime}\right)
$$

with $\hat{\sigma}_{i}$ a short-distance partonic cross section and $\phi_{i / H}$ the distribution of parton $i$ in hadron $H$.

I will begin with a review of three basic evolution equations, which govern the behavior of parton distributions in three limits of particular physical interest, and which are commonly referred to as the DGLAP, BFKL and Sudakov equations. Of these, the DGLAP is the beststudied, and controls the standard evolution of parton distributions in $Q^{2}$ for values of $x$ for which neither $\alpha_{s} \ln x$ nor $\alpha_{s} \ln (1-x)$ is a large number. The BFKL equation governs the behavior of parton distributions at small $x$ and fixed $Q^{2}$ and of amplitudes near the forward direction. Finally, the Sudakov evolution equation describes the behavior of distributions and amplitudes in the elastic limit $(x \rightarrow 1$ for deep-inelastic scattering). These limits are illustrated schematically in Fig. 1.

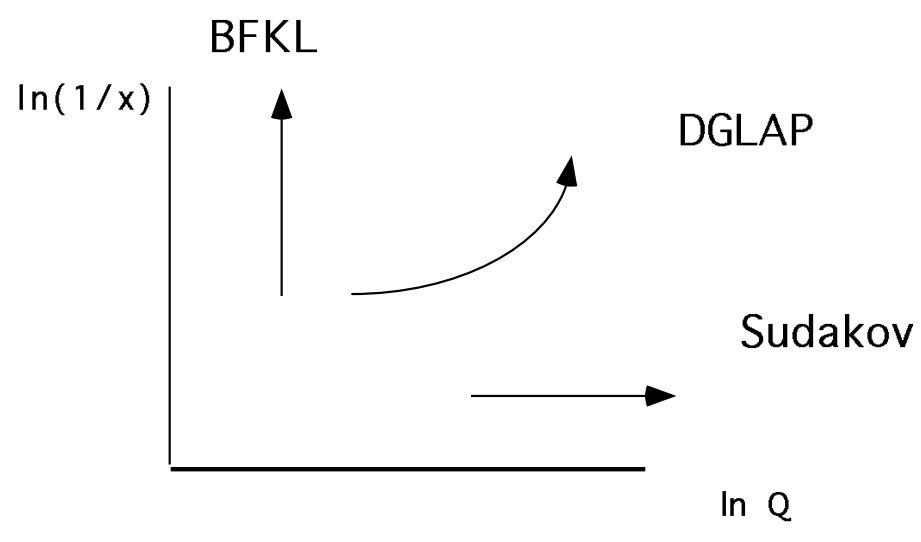

Figure 1: Actions of evolution equations.

Each of these evolution equations is associated with the ladder structure shown in Fig. 2, in which parton $j$, with momentum $p$ "evolves" into parton $i$, of momentum $x p$, in the process emitting $N$ quanta. In perturbation theory, the distribution of parton $i$ in parton $j$ may be thought of as a sum over $N$,

$$
\phi_{i / j}\left(x, q_{T}\right) \sim \sum_{N} \phi_{i / j}^{(N)}
$$




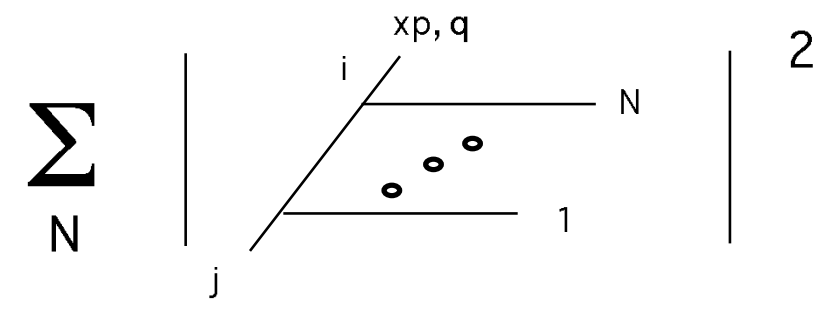

Figure 2: General ladder structure.

\subsection{DGLAP}

The basic evolution equation for perturbative QCD, originated by Dokshitser, Gribov, Lipatov, Altarelli and Parisi [四, has its origin in the description of deeply inelastic scattering (DIS). The factorization theorem for any DIS structure function $F$ in hadron $H$ is

$$
F^{(H)}\left(x, Q^{2}\right)=\int_{x}^{1} \frac{d x^{\prime}}{x^{\prime}} C_{j}\left(x / x^{\prime}\right) \phi_{j / H}\left(x, Q^{2}\right),
$$

with perturbative short-distance functions $C_{i}$ and nonperturbative parton distribution(s) $\phi$. Although $\phi$ is not perturbatively calculable, its $Q^{2}$ dependence is, in terms of kernels, or splitting functions, $P_{i j}$, through the DGLAP evolution equation

$$
\frac{d}{d \ln Q^{2}} \phi_{i / H}\left(x, Q^{2}\right)=\sum_{j=f, \bar{f}, G} \int_{x}^{1} \frac{d \xi}{\xi} P_{i j}(x / \xi) \phi_{j / H}\left(\xi, Q^{2}\right) .
$$

The universality of the distributions allow us to derive predictions for hadronic collisions from parton distributions derived (primarily) from DIS. The $P_{i j}$ themselves are known up to two loops.

In general, the DGLAP equation is applicable when the evolution is generated by the emission of quanta with strongly ordered transverse momenta $\left(k_{1 T} \ll k_{2 T} \ll \ldots \ll k_{N T}\right.$ in Fig. 2 , for instance). The integrals over these transverse momenta give logarithmic enhancements, which are organized by the DGLAP equation. There are other sources of logarithmic enhancement, however, already present as factors of $1 / x$ in the splitting function $P_{G G}(x)$. Such singular behavior produces logarithms of $x$, even in the absence of large transverse momentum enhancements. The BFKL equation, to which we now turn, summarizes the effects of these logs of $x$.

\section{$1.2 \quad$ BFKL}

The BFKL equation (Balitskii, Fadin, Kuraev and Lipatov [2]) was developed originally to resum $\operatorname{logs}$ of $s$ in the total hadronic cross section (see below). We begin our consideration of it here, however, with a generalization of DIS factorization (3), to a form that links the distributions in both longitudinal and transverse momenta [3],

$$
F\left(x, Q^{2}\right)=\int_{x}^{1} \frac{d x^{\prime}}{x^{\prime}} d^{2} k_{T} C\left(x / x^{\prime}, \mathbf{k}, Q^{2}\right) \mathcal{F}\left(x^{\prime}, \mathbf{k}\right)
$$

with $C\left(\xi, \mathbf{k}, Q^{2}\right)$ a new, transverse momentum-dependent short-distance function and $\mathcal{F}$ the corresponding parton distribution. In general, the gluon distribution tends to dominate at low 
$x$, and we shall restrict our discussion to $\mathcal{F}_{G}$. Eq. (5) is of particular interest when $x \rightarrow 0$, so that the mass of the final state in DIS, $(1-x) Q^{2} / x$, grows.

The BFKL equation, which describes the behavior of $\mathcal{F}_{G}$, may be written in many forms, one of the least intimidating of which is

$$
\frac{d}{d \ln x} \mathcal{F}_{G}(x, \mathbf{k})=\frac{3 \alpha_{s}}{\pi} \int \frac{d^{2} \mathbf{k}^{\prime}}{\left(\mathbf{k}^{\prime}-\mathbf{k}^{\prime}\right)^{2}}\left(K * \mathcal{F}_{G}\left(x, \mathbf{k}^{\prime}\right)\right),
$$

with a kernel defined by

$$
\begin{aligned}
K * \mathcal{F}_{G}\left(x, \mathbf{k}^{\prime}\right)= & \mathcal{F}_{G}\left(x, \mathbf{k}^{\prime}\right) \\
& -\left(\frac{\mathbf{k} \cdot \mathbf{k}^{\prime}}{2 \mathbf{k}^{\prime 2}}\right) \mathcal{F}_{G}(x, \mathbf{k}) .
\end{aligned}
$$

An excellent introduction to the BFKL equation may be found in the recent lectures of Del Duca [4].

The BFKL equation is somewhat harder to solve than the DGLAP equation, but it has many applications, including DIS for small $x$, semihard processes (minijet, heavy quark, etc.) in hadronic collisions, and color singlet exchange at $|t| \ll s$. Although the BFKL formalism has traditionally remained at leading logarithm in $x$, recent progress has been reported on generalizations that determine a two-loop kernel [5]. In addition, it is possible to develop a generalized evolution equation that interpolates between the DGLAP and BFKL equations at leading logarithm [6]. For this purpose, the angle of emission for soft gluons may be chosen as the primary evolution variable, reflecting an ordering of angles in sequential emission [7]. Control of such "coherence" effects in soft QCD radiation [8] also serves as an important ingredient in the construction of detailed models of QCD final states [9].

\subsection{Sudakov}

The other fundamental limit of evolution is $x \rightarrow 1$. In DIS, this corresponds to a low-mass final state with high energy. This "elastic" limit is illustrated in Fig. 3. In addition to soft radiation, a well-collimated jet of mass near $(1-x) Q^{2}$ emerges from the hard scattering $H$. Corresponding

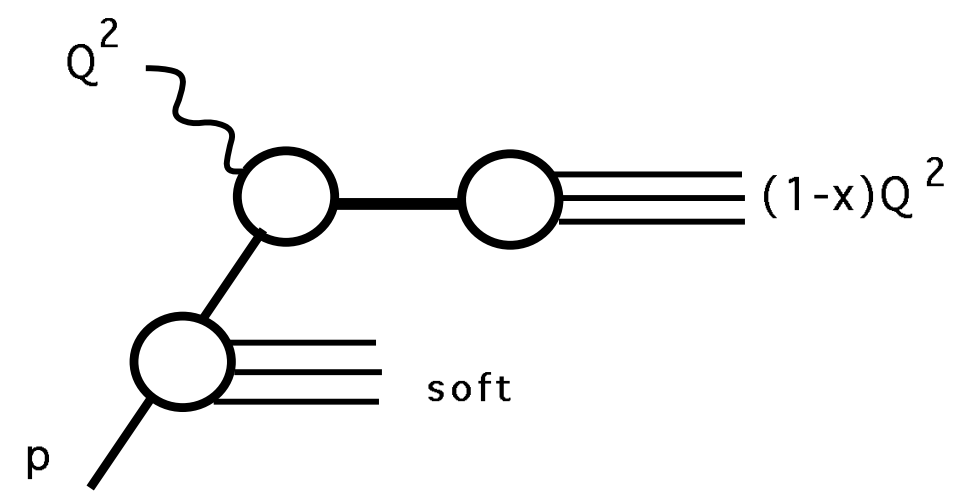

Figure 3: DIS as $x \rightarrow 1$.

to this physical situation, we may identify a factorized form for such a cross section,

$$
F\left(x, Q^{2}\right)=H(Q) \int_{x}^{1} d x^{\prime} J\left(\left(1-x^{\prime}\right) Q^{2}, Q\right) \phi\left(x^{\prime}, Q^{2}\right)
$$


with $J$ a universal function representing the hadronization of the jet (in this form certain nonleading logarithmic factors are suppressed). For $(1-x) Q^{2} \gg \Lambda^{2}, J$ is computable in perturbation theory. Given the factorization (8), the $x$ dependence of the jet may be determined from the Sudakov evolution equation [10],

$$
\begin{aligned}
& \left(\frac{\partial}{\partial \ln (1-x)}+\frac{1}{2} \beta(g) \frac{\partial}{\partial g}\right) J\left(\left(\quad 1-x^{\prime}\right) Q^{2}, Q\right)=\frac{1}{2} S\left(\alpha_{s}\right) J\left(\left(1-x^{\prime}\right) Q^{2}, Q\right) \\
& -\frac{1}{2} \int_{x}^{1} \frac{d y}{y} K_{J}(1-x / y) J\left((1-y) Q^{2}, Q\right),
\end{aligned}
$$

whose kernel $K_{J}$ is of the form of a plus distribution,

$$
K_{J}(1-z)=\left[\frac{\Gamma_{J}\left(\alpha_{s}\left((1-z)^{2} Q^{2}\right)\right.}{1-z}\right]_{+} .
$$

The function $\Gamma_{J}$ is a universal Sudakov anomalous dimension [11], related to the $\ln n$ dependence of the standard DGLAP anomalous dimensions $\gamma_{n}$ [12]. Thus, $\Gamma_{J}$ is also known to two loops. $S$ is a power series in $\alpha_{s}\left(Q^{2}\right)$. Sudakov evolution finds applications to jet event shapes, threshold corrections and transverse momentum distributions, some of which we shall review below.

\section{Resummation for Small $x$ and Diffractive Cross Sec- tions}

In this section, I will review a few of the prominent applications of the BFKL resummation of small- $x$ enhancements, beginning with DIS.

\section{$2.1 \quad$ DIS}

For DIS, the object of interest is the $k_{T}$-dependent gluon distribution, the solution to eq. (的) Eigenfunctions of the derivative may be found by direct substitution. The dominant power as $x \rightarrow 0$ specifies the growth of the gluon distribution. It is [2, 4]

$$
\mathcal{F}_{G}(x, \mathbf{k}) \sim x^{-\omega_{0}}
$$

times a power of $\mathbf{k}^{2}$, where

$$
\omega_{0}=\frac{4 N \alpha_{s}}{\pi} \ln 2
$$

with $N=3$ the number of colors. Since quark distributions mix with the gluon distribution, BFKL evolution suggests that the structure function of any hadron behaves as

$$
\begin{aligned}
F_{2}(x) & \sim \sum_{i} Q_{i}^{2} x \phi_{i}(x) \\
& \rightarrow x^{-\omega_{0}}
\end{aligned}
$$

which diverges as a power. This behavior is referred to as that of the "bare" pomeron. It is clear that such growth cannot be supported to arbitrarily small $x$, since if parton densities grow too large, they will begin to interfere or "shadow" each other. In technical terms, the assumption of 
low-density partons, which underlies each of the evolution equations above, fails for $x$ low enough [13].

HERA [14] has seen a growth in DIS structure functions at small $x$ which is consistent with the qualitative expectations of BFKL resummation. The DGLAP equation, however, based on $k_{T^{-}}$ordering, also results in growth for $x$ small, if not quite so dramatic. Untangling the physical content of the HERA data is a subject of great current interest [15.

\subsection{BFKL in Hadron-hadron Scattering}

The BFKL formalism [2] was originally developed to describe hadron-hadron scattering in QCD, both the total cross section and the closely-related Regge limit, $t$ fixed, $s \rightarrow \infty$. Its basic consequences for inclusive hadron-hadron scattering near the forward direction may by summarized by the following "translation" from DIS notation,

$$
\begin{aligned}
M_{\text {had }}^{2}=\frac{1-x}{x} Q^{2} & \rightarrow s \\
x^{-\omega_{0}} & \rightarrow s^{\omega_{0}} \\
F(x) & \rightarrow \sigma_{\text {tot }} \sim s^{\omega_{0}} .
\end{aligned}
$$

Note in particular that the total cross section grows as a power, corresponding to the $x \rightarrow 0$ behavior of $F_{2}(x)$ in DIS. Again, a rise in the total cross section for hadron-hadron scattering has long been seen at high energy, but an uninterrupted power-law rise would violate unitarity, as embodied in the Froissart bound. The "bare" pomeron identified above therefore cannot be the final answer. I shall return to recent progress on this question below.

It is of some interest to sketch the momentum-space configurations that give rise to the BFKL pomeron in hadron-hadron scattering. These are illustrated by the ladder diagrams in Fig. 4, where the vertices $C_{i}$ summarize contributions not only from the diagrams explicitly shown, but also from non-ladder diagrams of the same order that are important in gauge theories [2]. These

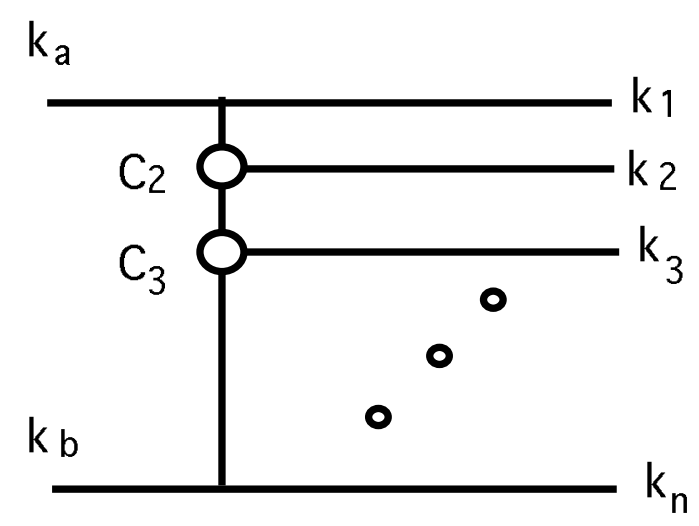

Figure 4: BFKL ladders.

vertices are related to the kernel in eq. (6) by $C_{i}^{2} \sim K$. As above, all lines in the ladder represent gluons. The growth of the cross section results from the production of many particles, whose momenta are conveniently parameterized in components parallel to the incoming momenta $k_{a}$ and $k_{b}$, and transverse components,

$$
k_{i}=\alpha_{i} k_{a}+\beta_{i} k_{b}+k_{i T} .
$$


The BFKL pomeron resums logarithms that result from configurations in which the rapidities $y_{i} \sim \ln \left(\alpha_{i} / \beta_{i}\right)$, are strongly ordered, but the transverse momenta are all of the same order as the momentum transfer,

$$
\begin{aligned}
\alpha_{1} \gg \alpha_{2} & \gg \cdots \gg \alpha_{n} \\
\beta_{1} \ll \beta_{2} & \ll \cdots \ll \beta_{n} \\
k_{i T} & \sim k_{j T} .
\end{aligned}
$$

It is the lack of ordering in transverse momentum that distinguishes BFKL evolution. Studies show that kinematic signs of this evolution should be present in final states [16], and data from the Tevatron and HERA are being closely scrutinized for evidence of these effects.

\subsection{Soft and Hard Diffraction}

Ampitudes for soft and hard diffraction are illustrated in Fig. 5. In 5a, the lines in both the cut and uncut ladder are ordered in rapidity, just as in Fig. 4. Only the cut lines, closer in rapidity

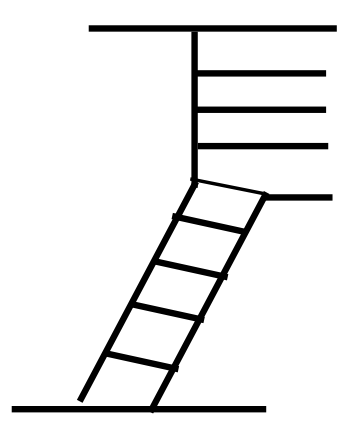

(a)

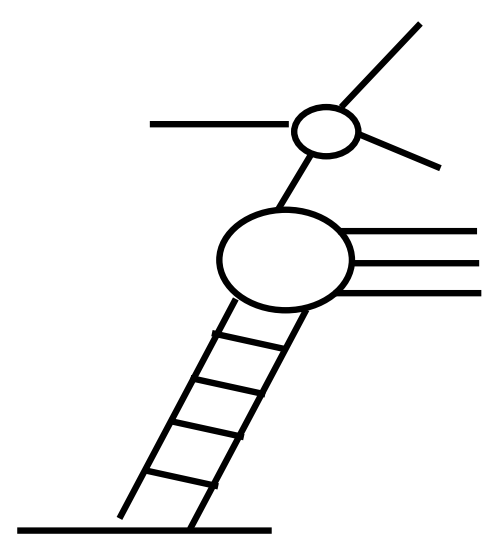

(b)

Figure 5: Schematic representations of soft (a) and hard (b) diffraction.

to the incoming top line appear in the final state, however, leading to a "gap" in rapidity, and an apparent "diffractive excitation" of the top line. The uncut ladder, a virtual pomeron, acts very much like a virtual elementary particle. Such events have been seen for some time, with particles that emerge into the final state generally at low transverse momentum. More recently, however, events have been seen that have both rapidity gaps and high momentum transfers [17. A possible mechanism is illustrated in Fig. 5b, in which the incoming top line undergoes a hard scattering from a "parton" of the pomeron. This idea 18 suggests that it might make sense to talk about the distributions of partons in pomerons. Such a formalism would be useful if these cross sections enjoy the universality and incoherence properties that underly normal factorization for inclusive cross sections [19]. Beyond this, the concept of rapidity gaps as a sign of color singlet exchange, even at large momentum transfer [20] is an attractive concept.

As noted above, the BFKL formalism treats leading logarihms of $s$. For singlest exchange, these are single logarithms per loop, $\alpha_{s}^{n} \ln ^{n} s$. More generally, elastic scattering of, say, highenergy quarks may involve both singlet and octet exchange

$$
A=A_{8} T_{8}+A_{1} T_{1},
$$


with the $T_{i}$ tensors in the color space. Supposing that $t$ is large, but $|t| \ll s$, it is possible to study the leading logarithms assoicated with soft gluon exchange according to Sudakov evolution methods [21]. The results for the $A_{i}$, neglecting for simplicity the effects of the running coupling, are of the schematic form 22, 23]

$$
A_{8} \sim e^{-\ln s \ln t}, A_{1} \sim e^{-\ln ^{2} t}
$$

This is consistent with BFKL resummation of $\operatorname{logs}$ of $s$ in singlet exchange at fixed $t$. For octet exchange, it is equivalent to a "Reggeized" gluon behavior [2]. These results are also consistent with the observation of rapidity gaps in hard scattering at high energy, and with their connection to singlet exchange. They are also relevant to a very different process, near-forward hard elastic scattering [24], in which an observed behavior of $t^{-8}$ may be a sign of triple pomeron exchange between valence quarks [23].

\subsection{Taming the Bare Pomeron}

As noted above, the bare pomeron violates the Froissart bound. Considerable effort recently has gone into remedying this defect, and to creating a formalism that reduces to the BFKL analysis for moderate $s$, but which has built-in unitarity. Here, I shall mention only a few examples. Lipatov has developed such a scheme, based on adding a subset of nonleading logarithms in $s$ and employing an expansion in $1 / N$, with $N$ the number of colors. In this scheme, the pomeron is a sort of bound state of Reggeized gluons [25]. Adding more such gluons, the problem reduces to finding the spectrum of a two-dimensional field theory in impact parameter space. Recently, Faddeev and Korchemsky [26] have shown that the resulting model is "solvable" in the sense of possessing infinite numbers of conservation laws. They do not, however, explicitly "solve" it.

Another approach toward deciphering the BFKL pomeron involves studying a model in which it arises self-consistently in perturbation theory, the scattering of bound states of heavy quarks [27. Although not yet realistic, such models allows a quite detailed study of soft gluon dynamics at large $N$ and leading logarithm.

\section{Resummation at the edge of phase space}

I now turn to applications of Sudakov resummation, beginning with a typical example from event shapes, the thrust.

\subsection{Thrust}

The thrust in $\mathrm{e}^{+} \mathrm{e}^{-}$annihilation is defined by

$$
T=\max _{\hat{n}} \frac{1}{Q} \sum_{i}\left|\vec{p}_{i} \cdot \hat{n}\right|,
$$

with the sum over all particles in the final state, and with a maximum taken over all unit vectors $\vec{n}$. In perturbation theory, the fixed-thrust cross section diverges at $T=1$, which is the elastic limit of two lightlike back-to-back jets. A measure of this divergence is given at one loop by

$$
\frac{1}{\sigma_{0}} \int_{0}^{T} d T^{\prime} \frac{d \sigma}{d T^{\prime}} \sim 1-\frac{\alpha_{s}}{\pi} C_{F} \ln ^{2}(1-T) .
$$


If the two jets have masses $p_{i}^{2} \sim(1 / 2)\left(1-x_{i}\right) Q^{2}$, then near the edge of phase space $x_{1}=x_{2}=1$, we have

$$
T \sim \frac{1}{2}\left[\left(1-x_{1}\right)+\left(1-x_{2}\right)\right] .
$$

In the same region, however, the cross section (simplified by neglecting certain nonleading corrections) factors into the product of the jet functions described above,

$$
\begin{aligned}
\frac{1}{\sigma_{0}} \frac{d \sigma}{d T^{\prime}} \sim \int d x_{1} d x_{2} \delta\left(1-T-\frac{1}{2}\left(2-x_{1}-x_{2}\right)\right) \\
\times J_{1}\left(\left(1-x_{1}\right) Q^{2}, Q\right) J_{2}\left(\left(1-x_{2}\right) Q^{2}, Q\right)
\end{aligned}
$$

each of which satisfies a Sudakov evolution equation, whose solution gives

$$
\begin{array}{rl}
\int d x e^{-\nu(1-x) Q^{2} / 2} & J \quad\left((1-x) Q^{2}, Q\right) \\
\sim & \exp \left[-\int_{0}^{1} \frac{d u}{u}\left(1-e^{-u \nu}\right) \int_{u^{2} Q^{2}}^{u Q^{2}} \frac{d \mu^{2}}{\mu^{2}} \Gamma_{J}\left(\alpha_{s}\left(\mu^{2}\right)\right)\right] .
\end{array}
$$

This result [28], and its analog for the transverse momentum distribution in Drell-Yan and $\mathrm{Z}_{0}$ production [29], are very helpful in understanding high energy data.

\subsection{Threshold Resummation}

Another important application of Sudakov resummation is to high-mass Drell-Yan, top and other cross sections where the parton distributions are falling rapidly at threshold. The simplest example is the inclusive Drell-Yan cross section,

$$
\frac{d \sigma}{d Q^{2}}=\sigma_{0} \sum_{q} \int_{\tau}^{1} d z \Phi_{q \bar{q}}(z) \omega_{q \bar{q}}\left(z, \alpha_{s}\left(Q^{2}\right)\right),
$$

where the partonic flux is given by

$$
\Phi_{q \bar{q}}(z)=\int_{0}^{1} \frac{d x_{a} d x_{b}}{x_{a} x_{b}} \delta\left(1-\tau /\left(z x_{a} x_{b}\right)\right) Q_{q}^{2} \phi_{q}\left(x_{a}, Q^{2}\right) \phi_{\bar{q}}\left(x_{b}, Q^{2}\right)
$$

with $Q_{q}$ the quark charge in units of electron charge and with $\sigma_{0}=4 \pi \alpha^{2} / 3 N Q^{2} s$ the Born cross section for $Q_{q}=1$. Here the overall partonic invariant is $\hat{s}=Q^{2} / z$, with threshold at $z=1$.

Specializing to DIS scheme, initial state radiation from the incoming quark pair in Drell-Yan cancels against initial state radiation in the DIS structure function, illustrated in Fig. 3 above. The (infrared safe) radiation from the outgoing quark jet in DIS, however, is not compensated. But since, as indicated above, the $x \rightarrow 1$ behavior of this jet can be computed, we can give an explicit form for the hard-scattering cross section $\omega_{q \bar{q}}$ in eq. (24) that includes all $\ln ^{n}(1-x)$ behavior in this limit. Schematically, it is of the form

$$
\omega_{q \bar{q}}\left(z, \alpha_{s}\left(Q^{2}\right)\right)=\delta(1-z)-\left[\mathrm{e}^{E}\left(1-z, \alpha_{s}\right) F(1-z)\right]_{+},
$$

where the function $F(1-z)$ is given by [30]

$$
F(1-z)=\frac{1}{\pi} \sin \left(\pi P_{1}\right) \Gamma\left(1+P_{1}\right)+\ldots
$$


with $P_{1}$ the derivative of the exponent $E$ with respect to $\ln (1-z)$,

$$
P_{1}\left(1-z, \alpha_{s}\right)=\frac{d}{d \ln (1-z)} E\left(1-z, \alpha_{s}\right) .
$$

$E\left(1-z, \alpha_{s}\right)$ is itself of the moment form,

$$
E\left(n, \alpha_{s}\right)=\int_{0}^{1} d x\left(\frac{x^{n-1}-1}{1-x}\right) \int_{(1-x)^{2} Q^{2}}^{(1-x) Q^{2}} \frac{d \mu^{2}}{\mu^{2}} g_{1}\left(\alpha_{s}\left(\mu^{2}\right)\right)+\ldots
$$

where $g_{1}$ is closely related to the jet anomalous dimension $\Gamma_{J}$, discussed above,

$$
g_{1}\left(\alpha_{s}\right)=2 C_{F} \frac{\alpha_{s}}{\pi}+\ldots=2 \Gamma_{J}\left(\alpha_{s}\right)+\ldots
$$

Given the form of $E$, we expect the effect of these corrections to be positive, and to raise the cross section above low order predictions. This expectation has been borne out by explicit calculations in leading-logarithm approximation for the DY process [31].

We have heard elsewhere at this conference that one-loop QCD predictions fall short of the very high- $E_{T}$ jet cross section measured by CDF. This cross section is of the same general form as above, given schematically by

$$
\left.\frac{d \sigma_{1 J}}{d E_{T}}\right|_{y=0}=\int_{2 E_{T} / \sqrt{s}}^{1} \Phi(z) \frac{d \hat{\sigma}_{1 J}}{d E_{T}}(\hat{s}=z s) d z .
$$

It is natural to ask whether this effect might be due to resummable soft gluon effects. Now we must note that in jet production final-state as well as initial state interactions may be important. Nevertheless, final-state interactions cancel in any cross section with finite angular and energy resolutions, leaving the same, universal initial-state corrections as in DY. It will be of great

interest to test these ideas in the coming months, since an excess of high-energy jets relative to QCD predictions could indicate profound new physics.

\subsection{Regulated Resummation}

Eq. (29) for the resummed exponent has a problem at small $1-x$, where the perturbative running coupling may diverge. To make sense of (29), one may use a cutoff in $x$ or $k_{T}^{2}$. Another possibility, following the example of ref. [32], is to define the integral as a principal value [33]. There is no special status for such a definition, but it enables us to express $E$ explicitly in terms of special functions, to be specific as a series of exponential integrals. In fact, any such definition of the perturbative expansion can be made only at the price of adding new nonperturbative parameters to the theory, as

$$
E_{N P}=\sum_{n \geq 1} \frac{A_{n}}{Q^{n}} .
$$

Cutoff integrals are probably numerically simpler. A sample application to resummed leading logarithms was studied in [3] for the Drell-Yan process,

$$
\int_{0}^{1} d x \frac{x^{n-1}-1}{1-x} \rightarrow \int_{0}^{1-\lambda / \Lambda} d x \frac{x^{n-1}-1}{1-x}
$$


Order by order, dependence on $\lambda$ is higher twist. For large enough $Q^{2}$, a cutoff resummation of this form is relatively insensitive to $\lambda$, but grows with $\tau$ at fixed $Q^{2}$ 31. Studies of the behavior at fixed $s$ are currently underway.

Finally, we may note that a cutoff prescription was employed in 355 to define resummed perturbation theory for top production, with partonic cross sections of the form

$$
\sigma_{i j}\left(z, m_{t}^{2}, s_{0}\right)=\int_{s_{0}}^{s-2 m_{t} \sqrt{s}} d s_{4} e^{\bar{E}\left(s_{4}\right)} \frac{d \sigma_{i j}^{(0)}}{d s_{4}}
$$

where $s_{4}=0$ at threshold, where $\bar{E}\left(s_{4}\right)$ is a resummed exponent related to (29) and where $\sigma_{i j}^{(0)}$ is the Born cross section.

\section{Infrared Renormalons and a New Source of Power Cor- rections}

Expanding the running coupling integrand in eq. (29) in terms of $\alpha_{s}\left(Q^{2}\right)$, we derive integrals of the form

$$
\left(-b_{2}\right)^{m} \alpha_{s}^{m} \int_{0}^{Q^{2}} \frac{d k_{T}^{2}}{Q^{2}} \ln ^{m}\left(\frac{k_{T}^{2}}{Q^{2}}\right)=b_{2}^{m} \alpha_{s}^{m} m !,
$$

in which the singularity in the running coupling is reflected in factorial growth of expansion coefficients in perturbation theory. This phenomenon is commonly referred to as an "infrared renormalon" 36. A closer analysis shows that such behavior may be interpreted as an ambiguity in the perturbative expansion, which may be removed by modifying the expansion to make it convergent, while at the same time adding a new term of the form of eq. (32), $A_{N} / Q^{N}$, with $Q$ the momentum transfer. The classic case [32] is the total cross section in $\mathrm{e}^{+} \mathrm{e}^{-}$annihilation, where the nonperturbative term is of the form $A_{4} / Q^{4}$, with $A_{4}=\left\langle F^{2}\right\rangle_{0}$, the vacuum expectation value of the squared gluon field strength. A similar analysis for resummed jet shapes [37] and DrellYan cross sections [33] shows that their resummed exponents potentially have a much stronger nonperturbative behavior [38, 39],

$$
E=E_{\mathrm{PT}}^{(\mathrm{reg})}+\frac{\Lambda}{\delta Q} A,
$$

where $\delta=0$ in the elastic limit (infinitely collimated jets, for instance). As in the case of the total $\mathrm{e}^{+} \mathrm{e}^{-}$cross section, it is possible to find a field-theoretic analog to $A$, but in this case it is in terms of a field-strength, integrated over the classical paths of the relevant partons. For example, for a two-jet cross section, with directions are $p_{1}$ and $p_{2}$, we find [39]

$$
\begin{aligned}
\Lambda A=\langle 0| \overline{\mathrm{T}}( & \left.\Phi_{p_{1}}^{\dagger}(0, \infty)\left(\mathcal{F}_{0 p_{1}}^{\dagger}(0)-\mathcal{F}_{0 p_{2}}(0, \infty)\right) \Phi_{p_{2}}(0, \infty)\right) \\
& \times \mathrm{T}\left(\Phi_{p_{1}}^{\dagger}(0, \infty) \Phi_{p_{2}}(0, \infty)\right)|0\rangle
\end{aligned}
$$

This matrix element is gauge invariant, with the $\Phi$ 's defined in terms of "Wilson lines", and the operator $\mathcal{F}$ in terms of the field strength. The rather general form of the operator suggests that these new corrections, suppressed by a single power of the large momentum scale, may possess universality properties. The concept of universality in this context has already been given much study [39, 40], and its promise and limitations have been discussed in 41]. 


\section{Conclusions}

Soft-gluon resummation allows varied applications of quantum field theory that are relevant to QCD at current energies. One goal of this program is to achieve a new level of precision

by combining large corrections to all orders. It also affords potential insights into features of nonperturbative structure, through the high-order behavior of the perturbation series.

Regarding specific applications, resummed threshold corrections to very high energy jet cross sections urgently need more study, especially because they are relevant to signs of new physics. Also, studies of $-t \ll s$ hard elastic and jet cross sections are attractive for their relevance to hard pomeron and Sudakov effects and to the valence-quark structure of the nucleon. Finally, infrared renormalons from resummation imply the existence a new class of nonperturbative parameters that may be measured in, for instance, jet shape analysis, and which may shed new light on the perturbative/nonperturbative interface in QCD.

It is a pleasure to thank Lyndon Alvero, Harry Contopanagos, Gregory Korchemsky and Jack Smith for many helpful conversations and explanations. This work was supported in part by the National Science Foundation under grant PHY9309888.

\section{References}

[1] G. Altarelli and G. Parisi, Nucl. Phys. B126 (1977) 298;

V.N. Gribov and L.N. Lipatov, Sov. J. Nucl. Phys. 15 (1972) 438, 675;

Yu.L. Dokshitser, Sov. Phys. JETP 46 (1977) 641.

[2] É.A. Kuraev, L.N. Lipatov, V.S. Fadin, Sov. Phys. JETP 45 (1977) 199;

Ya.Ya. Balitskii and L.N. Lipatov, Sov. J. Nucl. Phys. 28 (1978) 822.

[3] A. Sen, Phys. Rev. D27 (1983) 2997;

S. Catani, M. Ciafaloni and F. Hautmann, Phys. Lett. B242 (1990) 97; Nucl. Phys. B366 (1991) 135; Phys. Lett. B307 (1993) 147;

J.C. Collins and R.K. Ellis, Nucl. Phys. B360 (1991) 3.

[4] V. Del Duca, DESY 95-023, Feb. 1995, hep-ph/9503226.

[5] V.S. Fadin and L.N. Lipatov, Nucl. Phys. B406 (1993) 259;

V.S. Fadin (Novosibirsk, IYF). BUDKERINP-94-103, Dec 1994;

A.R. White, Phys. Lett. B334 (1994) 87;

C. Coriano and A.R. White, ANL-HEP-PR-95-12, Mar 1995, hep-ph/9503294.

[6] G. Marchesini, Nucl. Phys. B445 (1995) 49.

[7] A.H. Mueller, Phys. Lett. B104 (1981) 161;

B.I. Ermolaev and V.S. Fadin, JETP. Lett. 33 (1981) 269;

A. Bassetto, M. Ciafaloni, G. Marchesini and A.H. Mueller, Nucl. Phys. B207 (1982) 189.

[8] Yu.L. Dokshitzer, V.A. Khoze, S.I. Troyan and A.H. Mueller, Rev. Mod. Phys. 60 (1988) 373 ;

M. Ciafaloni, Nucl. Phys. B296 (1988) 49;

S. Catani, F. Fiorani, G. Marchesini and G. Oriani, Nucl. Phys. B361 (1991) 645. 
[9] G. Marchesini and B.R. Webber, Nucl. Phys. B310 (1988) 461; B386 (1992) 215;

M.H. Seymour, at the 10th Topical Workshop on Proton-Antiproton Collider Physics, Fermilab, 1995; hep-ph/9506421, Jun 1995.

[10] G. Sterman, Nucl. Phys. B281 (1987) 310.

[11] J.C. Collins and D.E. Soper, Nucl. Phys. B193 (1981) 381; B197 (1982) 446;

A. Sen, Phys. Rev. D24 (1981) 3281;

G.P. Korchemsky and A.V. Radyushkin, Phys. Lett. B171 (1986) 459.

[12] A.H. Mueller, Phys. Rev. D20 (1979) 2037;

J. Kodaira and L. Trentadue, Phys. Lett. 112B (1982) 66; 123B (1983) 335.

[13] L.V. Gribov, E.M. Levin and M.G. Ryskin, Phys. Rep. 100 (1983) 1;

E.M. Levin and M.G. Ryskin, Phys. Rep. 189 (1990) 267;

E. Laenen, E. Levin and A.G. Shuvaev, Nucl. Phys. B419 (1994) 39;

J. Bartels, Z. Phys. C60 (1993) 471.

[14] I. Abt et al., H1 Collaboration, Nucl. Phys. B407 (1993) 515;

M. Derrick et al., ZEUS Collaboration, Phys. Lett. B316 (1993) 412.

[15] S. Catani, DFF 207/6/94, hep-ph/9406325;

R.K. Ellis, F. Hautmann and B.R. Webber, Fermilab-PUB-95/006-T, hep-ph/9501307;

R.D. Ball and S. Forte, Phys. Lett. B336 (1994) 77;

J. Kwieciński, A.D. Martin and P.J. Sutton, Durham preprint DTP/95/22, hep-ph/9503266.

[16] V. Del Duca and C.R. Schmidt, Phys. Rev. D49 (1994) 177, 4510; D51 (1995) 2150;

W.J. Stirling, Nucl. Phys. B423 (1994) 56;

K. Golec-Biernat, J. Kwieciński, A.D. Martin, P.J. Sutton, Phys. Lett. B335 (1994) 220.

[17] A. Brandt et al., UA8 Collaboration, Phys. Lett. B297 (1992) 417;

G. Abachi et al.. D0 Collaboration, Phys. Rev. Lett. 72 (1994) 2332;

M. Derrick et al., ZEUS Collaboration, Phys. Lett. B315 (1993) 481.

[18] G. Ingleman and P.E. Schlein, Phys. Lett. B152 (1985) 256;

E.L. Berger, J.C. Collins, D.E. Soper and G. Sterman, Nucl. Phys. B286 (1987) 704;

J. Bartels and M. Wusthoff, Z. Phys. C66 (1995) 157.

[19] J.C. Collins, L. Frankfurt and M. Strikman, Phys. Lett. B307 (1993) 161;

J.C. Collins, J. Huston, J. Pumplin, H. Weerts and J.J. Whitmore, Phys Rev. D51 (1995) 3182 .

[20] J.D. Bjorken, Phys. Rev. D47 (1992) 101;

Y. Dokshitser, V. Khoze and S. Troyan, in Physics in Collision VI, Proceedings of the International Conference, Chicago, IL, 1986, ed. M. Derrick (World Scientific, Singapore, 1987), p. 365.

[21] J. Botts and G. Sterman, Nucl. Phys. B325 (1989) 62.

[22] G.P. Korchemsky, Phys. Lett. B325 (1994) 459.

I.A. Korchemskaya and G.P. Korchemsky, Nucl. Phys. B437 (1995) 127. 
[23] M.G. Sotiropoulos and G. Sterman, Nucl. Phys. B419 (1994) 59; B425 (1994) 489.

[24] A. Donnachie and P.V. Landshoff, Phys. Lett. B123 (1983) 345; Nucl. Phys. B231 (1984) 189.

[25] L.N. Lipatov, JETP Lett. 59 (1994) 596.

[26] L.D. Faddeev and G.P. Korchemsky, Phys. Lett. B342 (1995) 311;

G.P. Korchemsky, ITP-SB-94-62, Jan. 1995, hep-ph/9501232.

[27] A.H. Mueller, Nucl. Phys. B415 (1994) 373; B437 (1995) 107;

A.H. Mueller and B. Patel, Nucl. Phys. B425 (1994) 471;

N.N. Nikolaev, B.G. Zakharov and V.R. Zoller, Phys. Lett. B328 (1994) 486.

[28] S. Catani, L. Trentadue, G. Turnock and B.R. Webber, Nucl. Phys. B407 (1993) 3.

[29] J.C. Collins, D.E. Soper and G. Sterman, Nucl. Phys. B250 (1985) 199;

G. Altarelli, R.K. Ellis, M. Greco and G. Martinelli, Nucl. Phys. B246 (1984) 12;

C.T.H. Davies and W.J. Stirling, Nucl. Phys. B244 (1984) 337;

C.T.H. Davies, B.R. Webber and W.J. Stirling, Nucl. Phys. B256 (1985) 413;

P.B. Arnold and R.P. Kauffman, Nucl. Phys. B349 (1991) 381;

G.A. Ladinsky and C.P. Yuan, Phys. Rev. 50 (1994) R4239.

[30] H. Contopanagos and G. Sterman, Nucl. Phys. B400 (1993) 211.

[31] D. Appell, P. Mackenzie and G. Sterman, Nucl. Phys. B309 (1988) 259.

[32] A.H. Mueller, Nucl. Phys. B250 (1985) 327; in QCD 20 Years Later, Aachen, 1992, ed. P.M. Zerwas and H.A. Kastrup (World Scientific, Singapore, 1993) v.1, p.162.

[33] H. Contopanagos and G. Sterman, Nucl. Phys. B419 (1994) 77.

[34] L. Alvero and H. Contopanagos, Nucl. Phys. B436 (1995) 184.

[35] E. Laenen, J. Smith and W.L. van Neerven, Nucl. Phys. B369 (1992) 543; Phys. Lett. B321 (1994) 254.

[36] G. 't Hooft, in The Whys Of Subnuclear Physics, Erice 1977, ed. A. Zichichi (Plenum, New York, 1977), p. 943;

B. Lautrup, Phys. Lett. 69B (1977) 109;

G. Parisi, Phys. Lett. 76B (1978) 65; Nucl. Phys. B150 (1979) 163; Phys. Rep. 49 (1979) 215

F. David, Nucl.Phys. B234 (1984) 237; Nucl.Phys. B263 (1986) 637;

D. Appell, P. Mackenzie and G. Sterman in Proceedings of the Storrs Meeting, Fourth Meeting of the Division of Particles and Fields, Storrs, CT, August 15-18, 1988, p. 567;

V.I. Zakharov, Nucl. Phys. B385 (1992) 452;

M. Beneke and V.I. Zakharov, Phys. Rev. Lett. 69 (1992) 2472.

[37] G. Sterman, in proceedings of the Workshop on Physics at Current Accelerators and Supercolliders, Argonne National Laboratory, June 2-5, 1993, ed. J.L. Hewett, A.R. White and D. Zeppenfeld (Argonne National Laboratory, 1993) ANL-HEP-CP-93-92, p. 589. 
[38] B.R. Webber, Phys. Lett. B339 (1994) 148;

A.V. Manohar and M.B. Wise, Phys. Lett. B344 (1995) 407.

[39] G.P. Korchemsky and G. Sterman, Nucl. Phys. B437 (1995) 415.

[40] Yu.L. Dokshitzer and B.R. Webber, Cavendish preprint CAVENDISH-HEP-95-2, March 1995, hep-ph/9504219;

R. Akhoury and V.I. Zakharov, Saclay preprint SACLAY-SPHT-95-043, April 1995, hep$\mathrm{ph} / 9504248$.

[41] G.P. Korchemsky and G. Sterman, Stony Brook preprint, ITP-SB-95-17, May 1995, hepph/9505391;

P. Nason and M.H. Seymour, CERN/INFN, Milano preprint CERN-TH/95-150, IFUM 507/FT, June, 1995, hep-ph/9506317;

M. Beneke and V.M. Braun, DESY/Michigan preprint, June, 1995, DESY 95-120, UM-TH95-17, hep-ph 9506452. 